\title{
On steady hydromagnetic flow of a radiating viscous fluid through a horizontal channel in a porous medium
}

\author{
C. Israel - Cookey ${ }^{1,+}$, V. B. Omubo - Pepple ${ }^{2}$, I. Tamunobereton-ari ${ }^{2}$ \\ ${ }^{1}$ Department of Mathematics \\ ${ }^{2}$ Department of Physics \\ Rivers State University of Science and Technology, Port Harcourt 500001, Nigeria \\ +E-mail: Israel-cookey.chigozie@ust.edu.ng; cookeyci@yahoo.com
}

\begin{abstract}
This paper investigates the combined effects of radiative heat transfer and a transverse magnetic field on steady flow of an electrically conducting optically thin fluid through a horizontal channel filled with porous medium and non- uniform temperatures at the walls. Closed - form analytical solutions are constructed for the problem. The results are discussed quantitatively with the aid of the dimensionless parameters entering the problem.
\end{abstract}

Keywords: steady hydromagnetic, horizontal channel, porous medium, radiative heat, optically thin

\section{INTRODUCTION}

The flow of fluids through porous media are encountered in a wide range of engineering and industrial applications such as in recovery or extraction of crude oil, geothermal systems, thermal insulation, heat exchangers, storage of nuclear wastes, packed bed catalytic reactors, atmospheric and oceanic circulations. Comprehensive literature on buoyancy induced flows can be found Nield and Bejan (2006).

The study of flow of electrically conducting fluid, the so-called magneto-hydrodynamics (MHD) has a lot of attention due to its diverse applications. In astrophysics and geophysics, it is applied to the study of stellar and solar structures, interstellar matter, and radio propagation through the ionosphere. In engineering, it finds its application in MHD pumps, MHD bearings, nuclear reactors, geothermal energy extraction and in boundary layer control in the field of aerodynamics. A survey of MHD studies could be found in Crammer and Pai (1973); Moreau (1990). For example, Raptis et al. (1982) analyzed the problem of hydromagnetic free convection flow through a porous medium between two parallel plates; while Kearsley (1994) studied problem of steady state Couette flow with viscous heating. Makinde and Osalusi (2006) considered a MHD steady flow in a channel with slip at permeable boundaries.
More recently, many researchers have focused attention on MHD applications where the operating temperatures are high. For example, at high temperatures attained in some engineering devices, gas can be ionized and so become electrically conducting. The ionized gas or plasma can be made to interact with the magnetic field and alter the heat and friction characteristics of the system. It is important to study the effect of the interaction of magnetic field on the temperature distribution and heat transfer when the fluid is not only electrically conducting but also when it is capable of emitting and absorbing thermal radiation. Heat transfer by thermal radiation is important when we are concerned with space technology applications and in power engineering. Thus, Grief et al. (1971) obtained an exact solution for the problem of laminar convective flow in a vertical heated channel within the optically thin limit of Cogley et al. (1968). Makinde and Mhone (2005) investigated the effect of thermal radiation on MHD oscillatory flow in a channel filled with saturated porous medium and non-uniform wall temperatures. Kumar et al. (2010) considered the problem of unsteady MHD periodic flow of viscous fluid through a planar channel in porous medium using perturbation techniques. Narahari (2010) studied the effects of thermal radiation and free convection currents on the unsteady Couette flow between two vertical parallel plates with constant heat flux at one boundary. Israel - Cookey and Nwaigwe (2010) considered unsteady MHD flow of a radiating fluid 
over a vertical moving heated porous plate with time - dependent suction.

In this present paper, we investigate the combined effects of thermal radiation and transverse magnetic field on steady flow of electrically conducting optically thin fluid through a horizontal channel filled with saturated porous medium and non-uniform wall temperatures.

\section{MATHEMATICAL ANALYSIS}

Consider the buoyancy induced steady flow of an electrically conducting optically thin fluid bounded by two horizontal plates filled with saturated porous medium under the influence of a transverse uniform magnetic field of strength $\mathbf{B}_{0}$ as shown in Fig. 1. The lower plate which is on $y=0$ is maintained at temperature $T=T_{0}$ and the upper plate at $y=h$ is maintained at temperature $T=T_{1}$. Assuming a Boussinesq incompressible fluid model and taking into consideration the radiative heat flux, the governing equations for the flow are given by

$$
\begin{gathered}
v \frac{\partial^{2} U}{\partial y^{2}}+g \beta_{T}\left(T-T_{0}\right)-\frac{v}{k} U-\frac{\sigma_{c} B_{0}^{2}}{\rho_{0}} U=0 \\
\frac{\kappa_{T}}{\rho_{0} c_{p}}\left(\frac{\partial^{2} T}{\partial y^{2}}-\frac{1}{\kappa_{T}} \frac{\partial q}{\partial y}\right)=0
\end{gathered}
$$

where $U$ is the axial velocity, $T$ the temperature, $g$ the gravitational acceleration, $q$ the radiative heat flux, $\beta_{T}$ the coefficient of thermal expansion, $v$ the kinematic viscosity, $\sigma_{c}$ the electric conductivity, $\rho_{0}$ the fluid density, $c_{p}$ the specific heat capacity at constant pressure, $\kappa_{T}$ the thermal conductivity and $k$ the permeability of the porous medium.

The boundary conditions are

$$
\begin{array}{ll}
U=0, T=T_{0} & \text { on } y=0 \\
U=0, T=T_{1} & \text { on } y=h
\end{array}
$$

We assume that the temperatures $T_{0}, T_{1}$ of the walls are high enough to induce radiative heat transfer. Following Cogley et al. (1968) and assuming that the fluid is optically thin with relatively low density, then

$$
\frac{\partial q}{\partial y}=4 \alpha^{2}\left(T-T_{0}\right)
$$

where $\alpha$ is the mean radiation absorption coefficient.

In order to simplify the problem, we introduce the following non - dimensional variables and parameters

$$
\begin{aligned}
& y=h \eta U=U_{0} u, \theta=\left(T-T_{0}\right) /\left(T_{1}-T_{0}\right), k=h^{2} \chi^{2}, M^{2}=\sigma_{c} B_{0}^{2} h^{2} /\left(\rho_{0} \nu\right), \\
& G r=g \beta_{T} h^{2}\left(T_{0}-T_{1}\right) /\left({ }_{0} U_{0}\right), F^{2}=4 \alpha^{2} h^{2} / \kappa_{T}
\end{aligned}
$$

where $U_{0}$ is the mean velocity.

Using Eqs. (4) and (5) the dimensionless equations governing the flow together with the boundary conditions can be written as

$$
\begin{aligned}
& \frac{\partial^{2} u}{\partial \eta^{2}}+G r \theta-\lambda^{2} u=0 \\
& \frac{\partial^{2} \theta}{\partial \eta^{2}}-F^{2} \theta=0 \\
& u=0, \theta=0 \text { on } \eta=0 \\
& u=0, \theta=1 \text { on } \eta=1
\end{aligned}
$$

Here $\lambda^{2}=M^{2}+\chi^{2}, M$ is the magnetic parameter, $\chi$ the porosity parameter, $G r$ is Grashof number, and $F$ the radiation parameter. The mathematical statement of the problem is now complete and embodies the solution of Eqs. (6) and (7) subject to conditions (8).

The problem posed in Eqs. (6) and (7) are coupled nonlinear partial differential equations. Closed - form solutions are herein deduced. We begin by solving the energy equation (7) since it is uncoupled and then advance a solution for the flow velocity. The solutions to the temperature, $\theta(\eta)$ and velocity, $u(\eta)$ profiles are given by

$$
\begin{gathered}
\theta(\eta)=\frac{\operatorname{Sinh}(F \eta)}{\operatorname{Sinh}(F)} \\
u(\eta)=\frac{G r}{\left(\lambda^{2}-F^{2}\right)}\left(\frac{\operatorname{Sinh}(F \eta)}{\operatorname{Sinh}(F)}-\frac{\operatorname{Sinh}(\lambda \eta)}{\operatorname{Sinh}(\lambda)}\right)
\end{gathered}
$$

Skin friction: Using Equation (10), the skin - friction or the shear stress at the upper wall of the channel in non -dimensional form, is given by 
Am. J. Sci. Ind. Res., 2010, 1(2): 303-308

$\tau=-\left.\frac{\partial u}{\partial \eta}\right|_{\eta=1}=-\frac{G r}{\lambda^{2}-F^{2}}\left(\frac{F \operatorname{Cos}(F \eta)}{\operatorname{Sin} \nprec F)}-\frac{\lambda \operatorname{Cos}((\lambda \lambda \eta)}{\operatorname{Sin}(\lambda)}\right)$

Nusselt Number: From the temperature profile (Equation (9)), the rate of heat transfer across the channel in non - dimensional form is given by

$$
N u=-\left.\frac{\partial \theta}{\partial \eta}\right|_{\eta=1}=-\frac{F \operatorname{Cosh}(F)}{\operatorname{Sinh}(F)}
$$

\section{RESULTS AND DISCUSSION}

In the preceding section, we have formulated and solved the problem of steady hydromagnetic flow of a radiating viscous fluid through a horizontal channel filled with porous materials. The complete expressions for the velocity, $u(\eta)$ and temperature, $\theta(\eta)$ profiles as well as the skin friction, $\tau$ and the heat transfer rate, $\mathrm{Nu}$ are given in Equations (9) (12). In order to understand the physical situation of the problem and hence the manifestations of the effects of the material parameters entering the problem, we have computed the numerical values of the velocity, temperature, skin friction and the rate of heat transfer. The results are presented in Figures 2 -5 and Tables $1-3$.

The velocity profile for various values of the material parameters are plotted in Figures $2-4$. It can be seen that the fluid velocity profile is parabolic with maximum magnitude along the channel centerline and minimum at the walls. It is interesting to note that, the fluid velocity of the fluid decreased with increases in the magnetic field, radiation and porosity parameters. Similarly, the magnitude of the skin friction decreased with increases in the material parameters (see Tables 1 and 2).

In Figure 5, we present the behaviour of the temperature profile, $\theta(\eta)$ for various values of the radiation parameter, $F$. It is observed that the temperature profile increases with minimum at the lower plate and maximum at the upper plate. However, a general decrease in the fluid temperature profile within the channel is observed with increase in the radiation parameter. From Table 3, it is observed that the effect of increasing radiation is to increase the magnitude of the rate of heat transfer (Nusselt number).
Table 1: Shear stress at the upper wall for $G r=1, \chi=0.2$ and various values of $M$ and $F$.

\begin{tabular}{|c|c|c|c|}
\hline $\boldsymbol{F}$ & $\mathbf{M = 1}$ & $\mathbf{M = 2}$ & $\boldsymbol{M}=\mathbf{3}$ \\
& $\tau$ & $\tau$ & $\tau$ \\
\hline 0.1 & 0.312094 & 0.268024 & 0.223485 \\
0.2 & 0.311490 & 0.267543 & 0.223123 \\
0.3 & 0.310491 & 0.266746 & 0.222523 \\
0.4 & 0.309108 & 0.265643 & 0.221693 \\
0.5 & 0.307356 & 0.264246 & 0.220640 \\
0.6 & 0.305254 & 0.262569 & 0.219377 \\
0.7 & 0.302823 & 0.260629 & 0.217914 \\
0.8 & 0.300088 & 0.258446 & 0.216268 \\
0.9 & 0.297075 & 0.256040 & 0.214452 \\
1.0 & 0.293811 & 0.253432 & 0.212484 \\
& \multicolumn{3}{|c}{} \\
\hline
\end{tabular}

Table 2: Shear stress at the upper wall for $G r=1, M=1$ and various values of $\chi$ and $F$.

\begin{tabular}{|c|c|c|c|}
\hline $\boldsymbol{F}$ & $\chi=0.2$ & $\chi=0.4$ & $\chi=0.6$ \\
& $\tau$ & $\tau$ & $\tau$ \\
\hline 0.1 & 0.312094 & 0.266624 & 0.221345 \\
0.2 & 0.311490 & 0.266146 & 0.220988 \\
0.3 & 0.310491 & 0.265356 & 0.220398 \\
0.4 & 0.309108 & 0.264262 & 0.219580 \\
0.5 & 0.307356 & 0.262875 & 0.218544 \\
0.6 & 0.305254 & 0.261212 & 0.217299 \\
0.7 & 0.302823 & 0.259287 & 0.215860 \\
0.8 & 0.300088 & 0.257121 & 0.214238 \\
0.9 & 0.297075 & 0.254734 & 0.212450 \\
1.0 & 0.293811 & 0.252147 & 0.210511 \\
& & & \\
\hline
\end{tabular}

Table 3: Rate of heat transfer, $\mathrm{Nu}$ for various values of radiation parameter, $F$.

\begin{tabular}{ccccc}
$\boldsymbol{F}$ & $\mathbf{0 . 1}$ & $\mathbf{0 . 3}$ & $\mathbf{0 . 5}$ & $\mathbf{0 . 9}$ \\
\hline$|N u|$ & 1.00333 & 1.02982 & 1.08198 & 1.25646
\end{tabular}


Am. J. Sci. Ind. Res., 2010, 1(2): 303-308

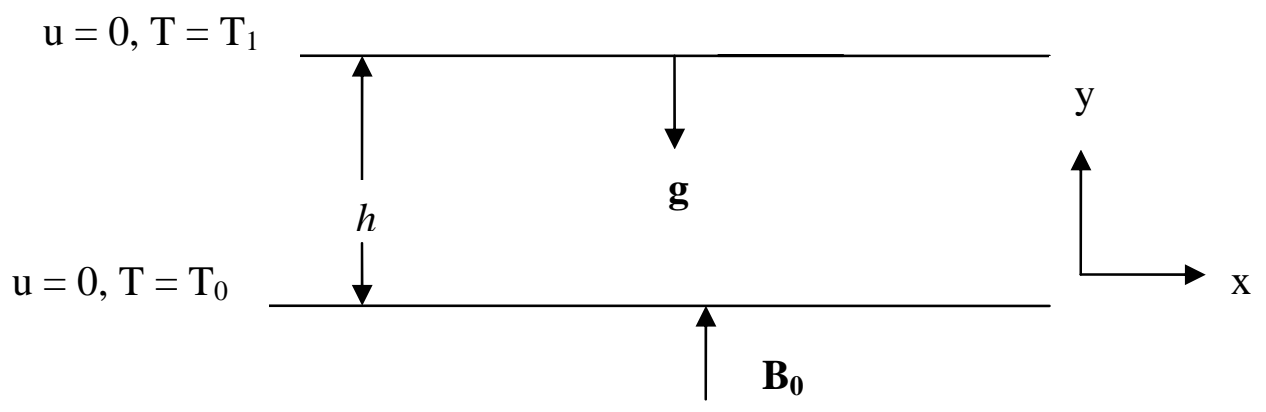

Fig 1: Geometry of the problem

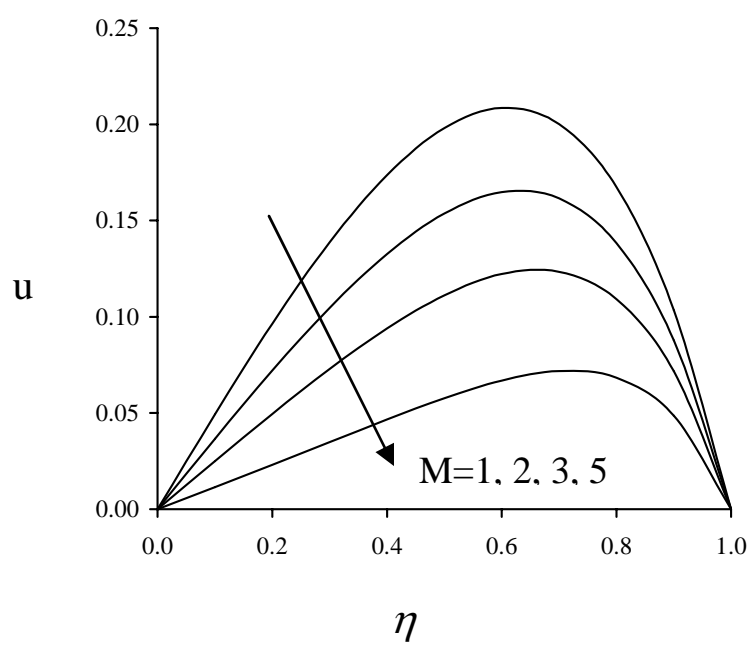

Figure 2: Velocity profiles as a function of $\eta$ for $\chi=0.2, F=2, G r=5$ and different values of magnetic parameter, $M$. 
Am. J. Sci. Ind. Res., 2010, 1(2): 303-308

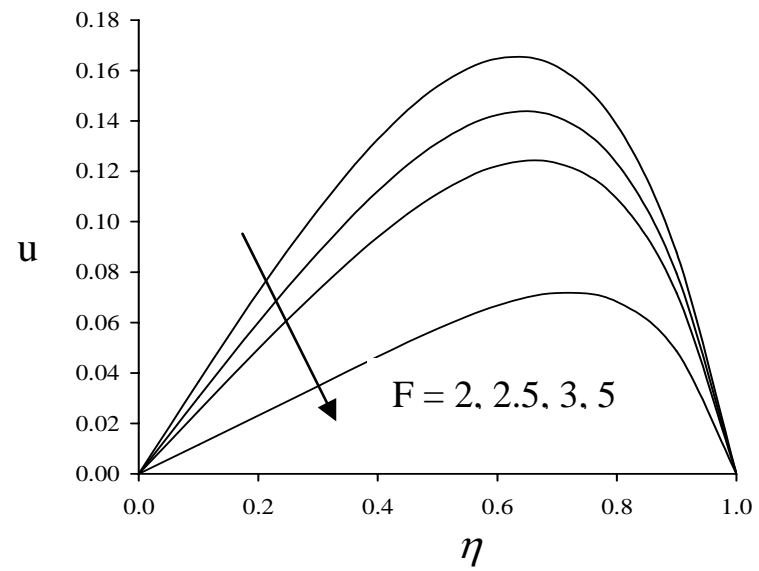

Fig 3: Velocity profiles as a function of $\eta$ for $\chi=0.2, M=2, G r=5$ and different values of radiation parameter, $F$.

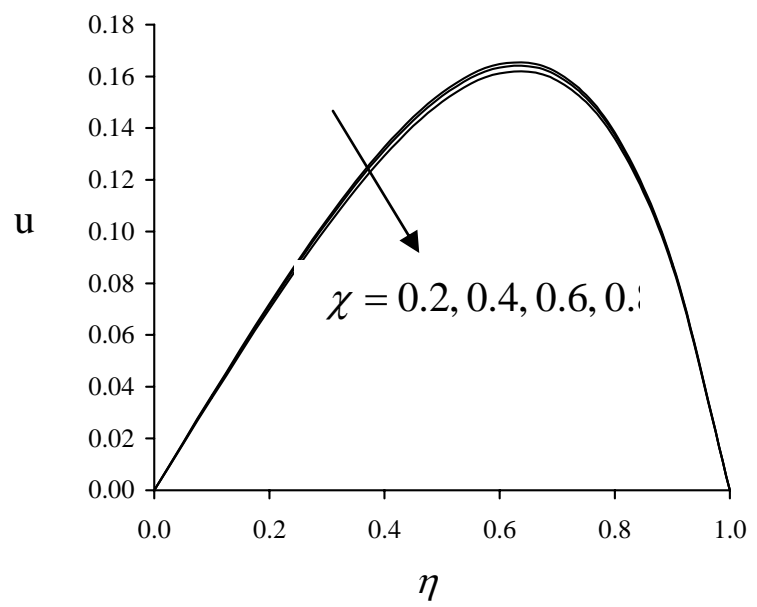

Figure 4: Velocity profiles as a function of $\eta$ for $F=2, M=2, G r=5$ and different values of porosity parameter, $\chi$. 


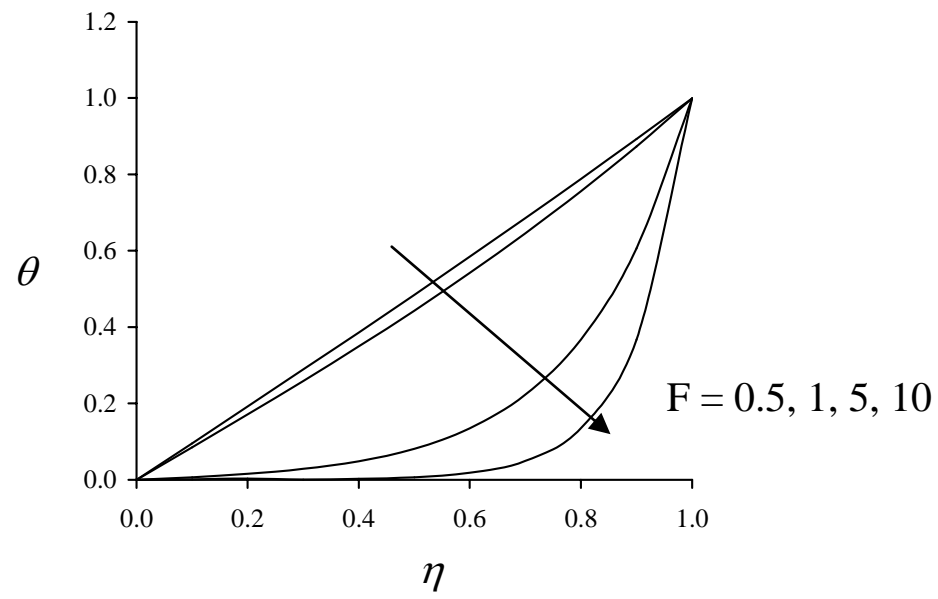

Fig 5: Temperature profiles as a function of $\eta$ for different values of the radiation parameter, $F$.

\section{CONCLUSION:}

In conclusion therefore, the problem of steady hydromagnetic flow of an optically thin viscous fluid through a horizontal channel filled with porous materials is affected by the magnetic parameter, radiation parameter as well as the porosity parameter. Generally, our results show that increasing magnetic field, radiation and porosity parameters reduced the velocity and temperature profiles as well as the shear stress at the wall while increasing radiation parameter causes an increase in the magnitude of the rate of heat transfer.

\section{ACKNOWLEDGEMENT:}

The authors are highly indebted to Mrs. G. Israel Cookey for excellent typesetting of the manuscript.

\section{REFERENCES}

Cogley, A. C. L., Vincenti, W. G., Gilles, E. S (1968), Differential approximation for radiative heat transfer in a non grey gas near equilibrium, Am. Inst. Aeronat. Astronaut. J 6: $551-553$.

Crammer, K., Pai, S. I (1973), Magnetofluid dynamics for engineers and applied physicists. McGraw-Hill Book Company.

Grief, R., Habib, I. S., Lin, J. C (1971), Laminar convection of a radiating gas in a vertical channel, $\mathrm{J}$ Fluid Mech. 46: $513-520$.
Israel - cookey, C., Nwaigwe, C., (2010), Unsteady MHD flow of a radiating fluid over a moving heated porous plate with time - dependent suction, Am. J. Sci. Ind. Res. 1(1): 88 - 95.

Kearsley, A. J (1994), A steady state model of Couette flow with viscous heating, Int. J. Engng Sci. 32: $179-186$.

Kumar, A., Varshney, C. L., Lal, S (2010), Perturbation technique to unsteady periodic flow of viscous fluid through a planar channel, J. Engng Tech. Research 2(4): $73-81$.

Makinde, O. D., Mhone, P. Y (2005), Heat transfer to MHD oscillatory flow in a channel filled with porous medium, Romanian J. Physics 50 (9-10): 931 - 938.

Makinde, O. D., Osalusi, E (2006), MHD steady flow in a channel with slip at permeable boundaries, Romanian J. Physics 51(3-4): 319 - 328.

Moreau, R (1990), Magnetohydrodynamics, Kluwer Academic Publishers.

Narahari, M (2010), Effects of thermal radiation and free convection currents on the unsteady Couette flow between two vertical parallel plates with constant heat flux at one boundary, WSEAS transactions on heat and mass transfer, 1(5): 21 - 30.

Raptis, A., Massias, C., Tzivanidis, G (1982), Hydromagnetic free convection flow through a porous medium between two parallel plates, Phys. Lett. 90(A): $288-289$. 